\title{
Pre-processing Technique for Wireless Capsule Endoscopy Image Enhancement
}

\author{
Rosdiana Shahril, Sabariah Baharun, AKM Muzahidul Islam \\ Malaysia-Japan International Institute of Technology (MJIIT), Universiti Teknologi Malaysia (UTM) \\ Kuala Lumpur Campus, Jalan Sultan Yahya Petra, 54100, Kuala Lumpur, Malaysia
}

\begin{tabular}{l} 
Article Info \\
\hline Article history: \\
Received Dec 11, 2015 \\
Revised Jun 3, 2016 \\
Accepted Jun 18, 2016
\end{tabular}

Keyword:

Anisotropic diffusion

Color contrast

Discrete cosine transform

Enhancement image

Hessian matrix

Wireless capsule endoscopy

\begin{abstract}
Wireless capsule endoscopy (WCE) is used to examine human digestive tract in order to detect abnormal areas. However, it has been a challenging task to detect an abnormal area such as bleeding due to poor quality and dark images of WCE. In this paper, pre-processing technique is introduced to ease classification of the bleeding area. Anisotropic contrast diffusion method is employed in our pre-processing technique as a contrast enhancement of the images. There is a drawback to the method proposed by B. Li earlier, in which the quality of WCE image is degraded when the number of iteration increases. To solve this problem, variance is employed in our proposed method. To further enhance WCE image, Discrete Cosine Transform is used with anisotropic contrast diffusion. Experimental results show that both proposed contrast enhancement algorithm and sharpening WCE image algorithm provide better performance compared with B. Li's algorithm since SDME and EBCM values are stable whenever the number of iterations increase. Moreover, the sharpness measurements using gradient and PSNR are both improved by $31.5 \%$ and $20.3 \%$, respectively.
\end{abstract}

Copyright (C) 2016 Institute of Advanced Engineering and Science. All rights reserved.

\section{Corresponding Author:}

Rosdiana Shahril,

Malaysia-Japan In ternational Institute of Technology (MJIIT),

Universiti Teknologi Malaysia (UTM),

54100 Kuala Lumpur.

Email: rosdianashahril@gmail.com

\section{INTRODUCTION}

Wireless capsule endoscopy (WCE) is a pill-shaped device, which captures the images while moving around the digestive tract to detect for any abnormalities. WCE consists of a light source, camera, radio transmitter and battery. The patient swallows the capsule like a pill, and then it captures the images in internal organ and sent out wirelessly to a special recorder which is attached to the patient's waist. This process continues until the end of battery's life. Finally, all images in the recorder are downloaded to inspect and compute by a physician. WCE images have very poor quality due of low transmission power of WCE device and bandwidth constraints.

Computer aided detection (CAD) system aims to reduce the burden and oversights of observations. The term of "computer aided detection system" is defined as a system or software that is assigned to recognize suspicious characteristics of the medical images before a physician inspects these images. The purpose is to reduce the miss-interpretation of the analysis of the images. Pre-processing techniques is required in CAD system as first step to enhance the quality of WCE images in order to detect the bleeding easily. Recently, researchers introduced various pre-processing techniques to improve CAD system.

The major contribution of the study is to design a pre-processing technique for computer aided detection (CAD) system. The aim is to detect the abnormalities such that classification process became easier. To detect the WCE images anisotropic diffusion proposed by [1] was further enhanced by using 
Hessian matrix, which gets a contrast description of one point in an image. However, this method has drawbacks where WCE images still has a noise and not sharp. Another drawback is quality of the image will be degrade if number of iteration goes up. Then, we solved this problem by introducing variance formula in their scheme [2]. To enhance the WCE image, Discrete Cosine Transform (DCT) is implemented with anisotropic contrast diffusion in order to get high quality WCE image which is more sharpness. Image sharpening is used to make fine details more clear or highlighted by amplify the high frequency of the image. Moreover, RGB is used in our proposed algorithm as color space to represent the details of WCE image. The rest of the paper is organized as follows. Section 2 presents the related works in pre-processing technique for CAD system and also mathematical background of this paper are briefly explained. Proposed method is described in Section 3 and followed by experimental results demonstrated in Section 4. Finally, concluding remarks of the paper is given in Section 5.

\section{RELATED WORKS}

Bleeding is used as an indication of some severe conditions and diseases such as vascular lesions, tumors and Crohn's disease. Common color space used is RGB which is contained three channels; red, green and blue channel. Another color spaces used are HIS, HSV, YCbCr and CIE Lab. HIS and HSV are the two most common visions and perception representation of points in RGB color space. Researchers introduced many pre-processing techniques. In [3], weight factor is used to identify bleeding by deriving features of bleeding region color. Six statistical parameters are introduced in [4] to extract the features from bleeding in spatial characteristics in HIS color space from the images. In [5], color spaces such as RGB, HSB and YUV are investigated to see which color space is able to disclose lesion structure and geometry, color and texture to determine and to be considered in their analysis.

Histogram based index image is used in [6] to extract color texture of bleeding. In [7], ratio of R to G pixel intensity and different statistical parameters are used to extract the bleeding. In [8], RGB and HIS are used as color spaces in extraction of bleeding features. They remove dark pixels from WCE images since they are difficult to be recognized by human eyes. In [9], CIE Lab color space is used as color spaces in their proposed techniques. Four functions are used in their scheme including parameters which depend on eigenvalues of Hessian and Laplacian to detect the bleeding region. In [10], histogram probability is used by calculating mean and standard deviation to extract color features of bleeding. In this proposed method, anisotropic diffusion also known as Perona-Malik diffusion, a very popular technique aiming to reduce image noise is employed. It is used to enhance WCE images by reducing noise without removing the significant parts of the image content. In [11], artificial fish-warm algorithm was used to enhance medical digital radiography (DR) image which is effective in eliminating noise and enhancing the detail with high effectiveness and robustness. Multi-wavelet transform and median filter is used in [12] to remove the impulse noise viewed as random noise from the blurred underwater image.

\subsection{Anisotropic Diffusion}

Perona-Malik model has introduced a new definition of scale-space and class algorithms using a diffusion process to avoid the blurring and also localization problems of linear diffusion filtering. Anisotropic diffusion method proposed by Perona-Malik is mathematically formulated as a diffusion process, and encourages intra-region smoothing in preference to smoothing across the boundaries. It is a very popular technique to reduce image noise, which is also used to enhance WCE images, by reducing noise without removing the significant parts of the image content. In filtering method, the estimation of the local image structure is driven by the knowledge on the statistics of the noise degradation and edge strengths [13],[14]. Perona-Malik model is outperformed compared to Canny edge detector which is edges persisted stable over a very long time even without using non-maxima suppression and hysteresis thresholding. Given the original image $u_{0}(x, y, t)$ and the smoothed versions comprise a family images $u(x, y, t)$ where $t$ is the amount of smoothing. Anisotropic diffusion of Perona-Malik is defined as [13]:

$$
u(t)=\operatorname{div}[g(x, y, t)|F u|]=g(x, y, t) \& u+\nabla_{g}, F_{u}
$$

where div is the divergence operator, $\nabla u=\left[L_{\alpha} u_{y}\right]$ is the spatial gradient, $\|\nabla u\|=\sqrt{u_{\alpha}^{2}+u_{\gamma}^{2}}$ is the gradient magnitude, and $\mathrm{g}(\mathrm{x}, \mathrm{y}, \mathrm{t})$ is a diffusion coefficient. $\mathrm{g}(\mathrm{x}, \mathrm{y}, \mathrm{t})$ controls the rate of diffusion. The isotropic heat diffusion equation, $\mathrm{u}_{\tau}=c \Delta \mathrm{s}$ is reduced if $\mathrm{g}(\mathrm{x}, \mathrm{y}, \mathrm{t})$ is a constant.

$$
g(x, y, t)=g(\|F w(x, y, t)\|)
$$



follows:

Discretize the equation (1) and 4 nearest-neighbors discretization was proposed by Perona-Malik as

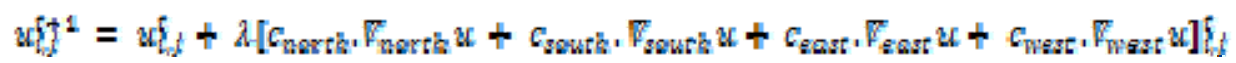

where, $\lambda \leq \frac{1}{4}$ for the numerical scheme to be stable and the symbol $\bar{\nabla}$ indicates nearest-neighbors differences,

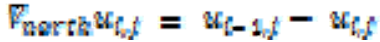

$$
\begin{aligned}
& F_{\text {souta }} v_{i j t} \equiv v_{i+1, j}-w_{i j} \\
& F_{\operatorname{eagt}} w_{\eta_{j}} \equiv w_{\eta_{j}+1}-w_{\eta_{j}}
\end{aligned}
$$

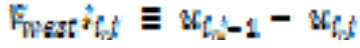
gradient:

Each iteration updates the conduction coefficients as a function of the magnitude of the brightness

$$
\begin{aligned}
& \text { Garthy }-g\left(F_{\text {norta }} \mathrm{w}_{\mathrm{g}} \mathrm{b}\right. \\
& c_{\text {sew: }}^{t}=g\left(\mid F_{\text {sawr }} w_{j} b\right. \\
& c_{\text {engt }}^{t}=g\left(\mid F_{e x g} d d\right) \\
& \sigma_{\text {Test }}^{5}=g\left(\mid F_{\text {mest }} w_{j} b\right)
\end{aligned}
$$

where, $\mathrm{g}$ has two function differences and defined as:

$$
g\left(\|x\| \tilde{L}=\exp \left\{-\left(\frac{F u}{R}\right)^{2}\right\}=\frac{1}{1+\left(\frac{R u}{R}\right)^{2}}\right.
$$

The parameter of constant $\mathrm{K}$ is a conductance parameter that influences the diffusion process which is separates forward (low contrast) from backward (high contrast) diffusion areas.

\subsection{Hessian matrix}

Hessian matrix is a square matrix of second-order partial derivatives of a function and it used as approach in this work to get a contrast description of one point in an image. Hessian matrix of one point in a gray image which is defined as follows [1]:

$$
R(x, y)=\left[\begin{array}{ll}
w_{x x} & w_{x y} \\
w_{y x} & w_{y y}
\end{array}\right]
$$

where, $u_{k x} u_{x y} u_{y x}$ and $b_{y y}$ are the second-order derivative of the image along direction of $\mathrm{x}, \mathrm{y}, \mathrm{xy}$ respectively and $u_{x y}=u_{y x} \cdot[1]$ has proposed a new concept of contrast as follows:

$$
\mathrm{s}(\mathrm{x}, \mathrm{y})=\mathrm{v}_{1}^{2}\left(\mathrm{x}, y^{2}\right)+\mathrm{v}_{2}^{2}\left(\mathrm{x}, y^{2}\right)
$$

where, $v_{1}$ and $v_{2}$ is a two eigen value of Hessian matrix and denoted by [15].

$$
\begin{aligned}
& v_{1}=\frac{1}{2}\left[\left(u_{x x}+v_{y y}\right)+\sqrt{\left(u_{x x}-u_{y y}\right)^{2}+4 w_{x y}{ }^{2}}\right] \\
& v_{2}=\frac{1}{2}\left[\left(u_{x x}+w_{y y}\right)-\sqrt{\left(u_{x x}-u_{y y}\right)^{2}+4 w_{x y}{ }^{2}}\right]
\end{aligned}
$$

Employing this contrast space, proposed by [1], change the original anisotropic diffusion into:

$$
u(t)=d w[g(c)|F c|]=g(c) \Delta c+\mathbb{F} g F c
$$




\subsection{Gaussian filter}

Gaussian filtering is used in the smoothing of an image to remove noise in WCE images. In our proposed method, two dimensional of Gaussian filter is used as a kernel to convolve with an image since working with image.

Output $=f^{*} z$, where $f$ is an image and $g$ is a kernel. Gaussian filter is defined as:

$$
G(x, y)=\frac{1}{2 \pi \sigma^{2}} e^{\frac{x^{2}+y^{2}}{2 \sigma^{2}}}
$$

where, $\boldsymbol{\sigma}$ is a standard deviation of the Gaussian distribution. It is accomplished by convolving between a kernel and image. The output image pixel is calculated by multiplying each kernel value by the corresponding input image pixel.

\section{THE PROPOSED METHOD}

Pre-processing technique is proposed in this paper and presents the step-by-step for WCE images enhancement. In the proposed method, contrast enhancement method is employed to make more contrast on the WCE images compared to these using the original concept introduced by B. Li [1]. B. Li's algorithm is based on anisotropic contrast diffusion and Hessian matrix. Here, variance formula is introduced to overcome the B. Li's weaknesses. In order to make the details of each image to be more visible, sharpening algorithm is proposed to ease the classification process of the abnormalities such as bleeding in WCE images [16].

\subsection{Contrast Image Enhancement}

In our proposed method, anisotropic contrast diffusion is employed to contrast the images due to low dark quality of the WCE images. It also able to make characteristics in a WCE image is more visible by human eyes also by computer machine.

Due to the weakness of B. Li's as explained in Section 1, thus variance is introduced in this proposed method to overcome the weakness. The variance $\left(\operatorname{Var}^{2}\right)$ is a measure of the spread of pixel values around the image mean. By using the variance, it estimates the contrast probability distribution of the image. It will give an idea how the pixel value spread in image. A small variance means that the data points tend to be very close to the mean while high variance means that the data points spread out around the mean and from each other. Therefore, it does not degrade the quality of the image. The variance formula $\left(\operatorname{Var}^{2}\right)$ is:

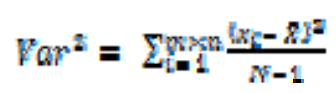

where, $\bar{X}$ is a mean of 4 nearest-neighbors, $\mathrm{N}=$ size of image $\mathrm{m} \times \mathrm{n}$ and $\mathrm{x}_{\mathrm{i}}$ is a pixel of image $i$.The following formula is used to calculate the mean of 4-nearest-neighbors, $\overline{\mathrm{x}}$ :

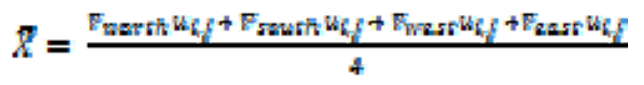

The Eq. (9) is revised by using standard variance, $\left(\operatorname{Var}^{2}\right)$ as follows:

$$
g(c)=\frac{1}{1+\left(\frac{\left.\mid \operatorname{son}^{2} x^{2}\right)^{2}}{x}\right)}
$$

In order to get more high quality WCE image which is sharper, DCT is used with anisotropic contrast diffusion where DCT is enabling to sharp WCE image and the details is explained in the next section.

\subsection{Sharpening Image Using Discrete Cosine Transform (DCT)}

A DCT can be used in image processing such as pattern recognition and filtering by computing the fast Fourier transform is developed. In WCE image sharpening, the idea is to use high frequency components that are extracted from the original images to highlight the invisible details. High frequency is used to determine or detect the edge information. Examples of such methods are Sobel, Canny, and unsharp masking but these methods are more sensitive to noise. Sharpening image is done, by restoring high frequency into original image. On the other hand, blurred image is produced by loss of high frequency. In our method, high 
frequency containing the fine details of the original image is extracted by using DCT then it is combined with the WCE original image to produce a new image with improved quality in visual appearance. The following mathematical model is assumed for input RGB image:

$$
w_{R, E}=z_{R, E}+n_{N_{i}, E}
$$

where $u, z$ and $n$ are the input noisy image, the noise free image and the noise component respectively in red, green and blue channel. In human eye, it is more sensitive to flickering of high spatial frequencies than low spatial frequencies. By using this concept, DCT is proposed in our scheme to amplify the image frequency to sharp the image. The DCT of a data sequence $u(x, y), x=0,1, \ldots,(M-1), y=0,1, \ldots,(N-1)$ is defined as [17]

$$
C_{d}(m, n)= \begin{cases}\frac{\sqrt{2}}{M} \sum_{m=1}^{M-1} \sum_{n=n}^{N-1} w(x, y), \quad m=0, n=0 \\ \frac{2}{M} \sum_{m=1}^{M=1} \sum_{n=1}^{M=1} w(x, y) \cos \frac{(2 x+1) m w}{2 M} \times \cos \frac{(2 y+1) n \pi}{2 N} \\ 1 \times m \times M-1,1 \times n \times N-1\end{cases}
$$

where $C_{d}(x, y)$ is the $d$-th DCT coefficient, $u(x, y)$ represents the image data, and $\mathrm{M}, \mathrm{N}$ is the width and length of $u(x, y)$. In this step, DCT is used to extract the high frequency in WCE image. Then, high frequency is applied to the original image to sharpen the WCE image as shown in Figure 1.

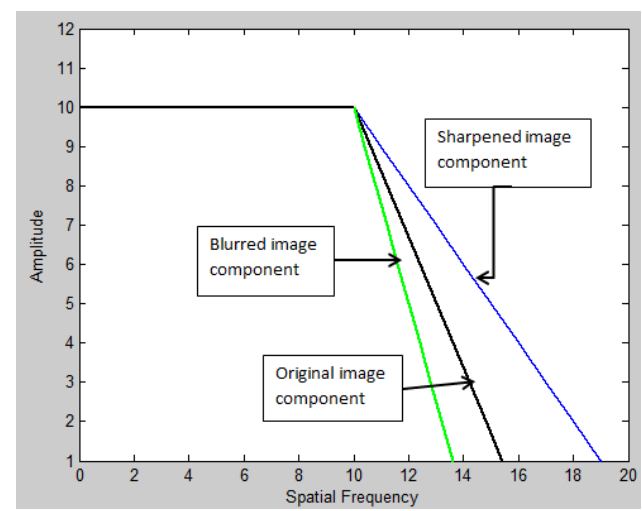

Figure 1. Component of original, sharpened and blurred image in frequency domain

Let $X$ be the sharp image, $I$ be the original image that is to be recovered and $Y$ be the high frequency, then their relation is represented using the following equation:

$$
X=I+Y
$$

This image is transformed into DCT blocks in the frequency domain. The first DCT coefficient top left in block DCT is $\mathrm{F}(0,0)$, where it represents the average intensity of the block. It is also known as the DC component or energy blocks. Other blocks of DCT coefficients are called AC coefficients. Generally, sharp spaces such as edges correspond to high frequency region while long unchanging spaces correspond to low frequency region [17],[18]. After combining the high frequency with original image, the image is transformed back into the spatial domain using the inverse cosine discrete transform (ICDT) which is defined by:

$$
w(x, y)=\frac{1}{2} c_{d}(0)+\sum_{p=1}^{M-1} \sum_{q=1}^{N-1} c_{\alpha} \cos \frac{\pi(2 m+1) p}{2 M} \cos \frac{\pi(2 n+1) q}{2 N}
$$

where $\mathrm{m}=0,1, \ldots,(\mathrm{M}-1)$ and $\mathrm{n}=0,1, \ldots,(\mathrm{N}-1)$. 
The following is the algorithm for the proposed method implementation:

Step 1: Read image

Step 2: Calculate Hessian matrix and get eigenvalues, $v_{1}$ and $v_{2}$

Step 3: Calculate $c=v_{1}^{2}+v_{2}^{2}$

Step 4: Start number of iteration for each channel, RGB

Step 4.1: Reduce noise using Gaussian filter, $G=$ Gaussian (c, $\left.\sigma^{*}\right)$

Step 4.2: Convolve the image between kernel (Gaussian filter), $I=G^{*} C$

Step 4.3: Apply the standard variance, $\sigma^{2}$

Step 4.4: Calculate the diffused image by using anisotropic Perona-Malik, $u=\operatorname{div}\left(g^{*} I\right)$

Step 5: Get $C_{\min }$ and $C_{\max }$

Step 6: The diffused result is transformed back to image space, Output $=\left[\left(c-c_{\min }\right) /\left(c_{\max }-s c_{\min }\right)\right] * 255$

Step 7: Apply the DCT, $C_{d}$

In this algorithm, $C_{\min }$ and $C_{\max }$ is obtained from $c=v_{1}^{2}+v_{2}^{2}$ where $C_{\min }$ and $C_{\max }$ is the minimum and maximum value of $c(x, y)$, respectively.

\section{EXPERIMENTAL RESULTS AND DISCUSSION}

Matlab R2011b is used in this experiment. Four images are used and the expected result of this paper is to produce high quality WCE images that are sharper and contrast images. In color image enhancement technique, it is very hard to analyze the quantitative effect, as there is no specific measurement to validate the quality of the color image. Here, comparison between B. Li's method and proposed method using variance (without sharpening using DCT) is presented in Figure 2.

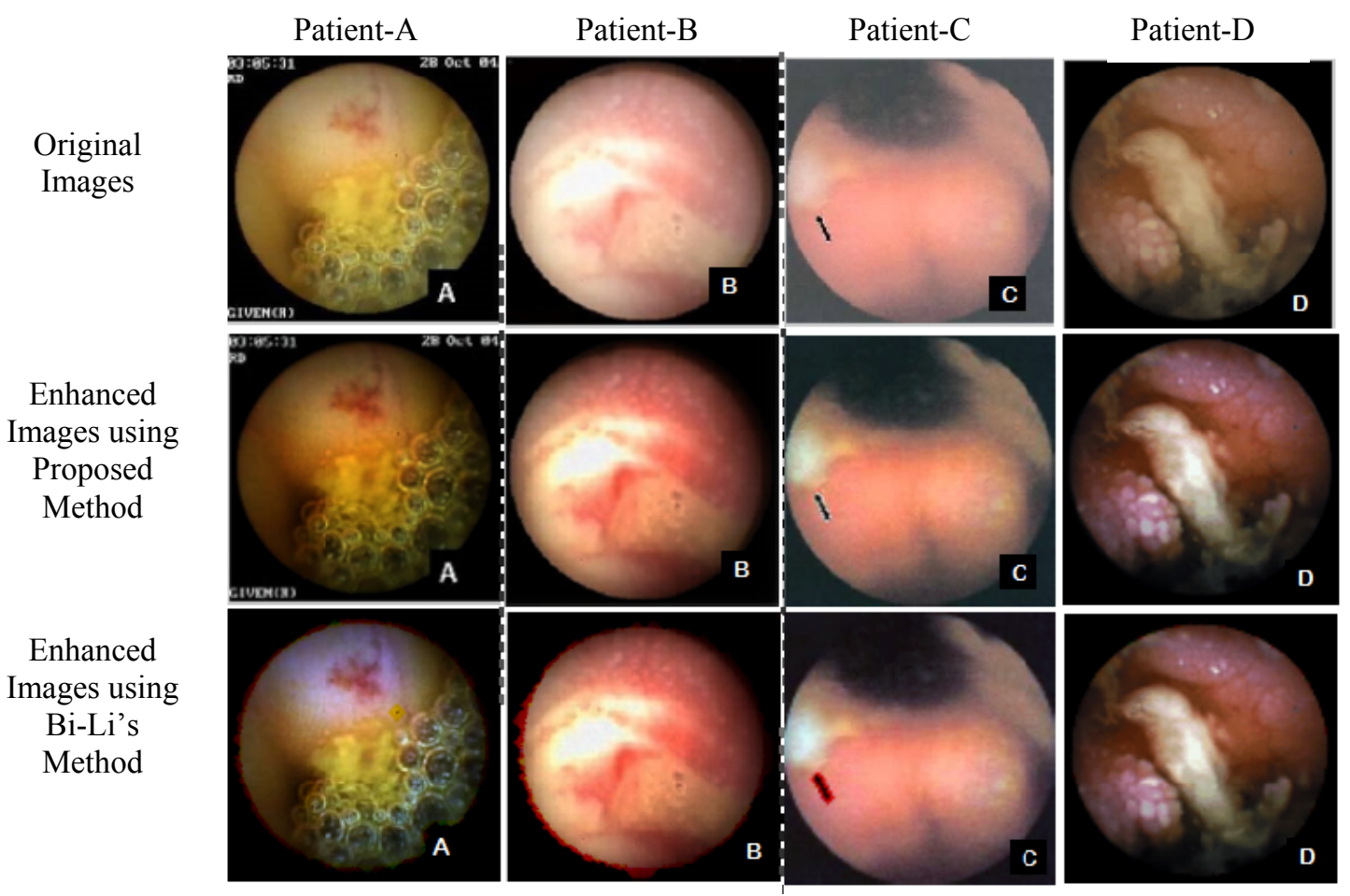

Figure 2. The original images compared to B. Li's and proposed method

Original images in this figure have poor quality and are vague. However, the enhanced image by the proposed method become much better and more contrast, and not degrading the image. This can be seen that eventhough B. Li's method do make some enhancement to the original but due to its limitation and some drawback as discussed in previouse section. The introduction of variance in B. Li's method proves to give better enhancement of the images.

Quality measure analysis on WCE images using Second Derivative like Measurement (SDME) and Edge Based Contrast (EBCM) as the performance estimation standard is considered. EBCM is very sensitive to contours or edges due to based human perception. EBCM is defined by [19],[20]. 


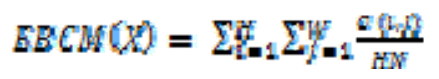

Contrast $c(i, j)$ for a pixel of image $\mathrm{X}$ located at $(i, j)$ is thus defined as

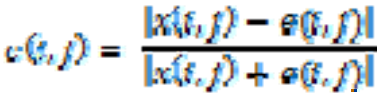

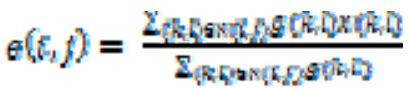

where, $g(k, l)$ is the edge value at pixel $(k, l)$ and $\mathbb{N}(h, \mathcal{H})$ is the neighboring pixels at $(i, j)$.

On the other hand, SDME divide an image into $k_{1} \times k_{2}$ blocks and then average values of the measure results of all block is calculated in the entire image. SDME is defined by [21]

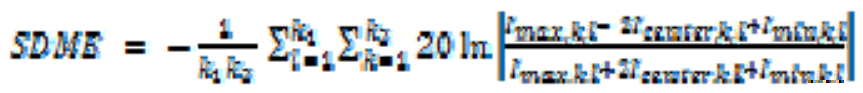

By using both SDME and EBCM, it is proved that the proposed method (black line) is better than B. Li's method (yellow line) as shown in Figure 3 and Figure 4. The higher value of SDME and EBCM means that the images have a better quality. It shows that variance is able to solve the weakness of B. Li's method whereby the quality of image will not degrade when the number of iteration is increased. Here the number of iteration is defined as the number of repetiting the process or iterating operations yielding result closer to the desired one.
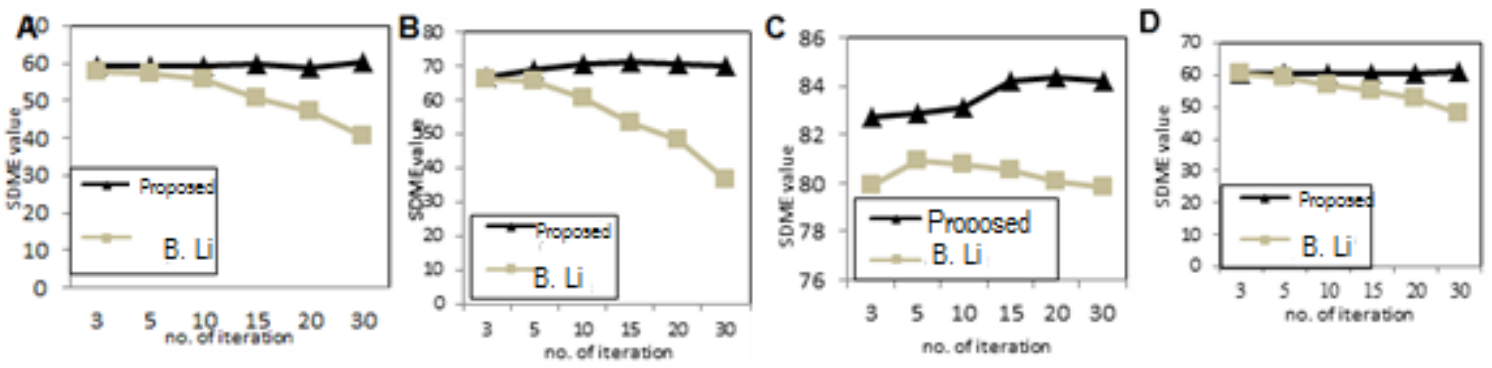

Figure 3. SDME measure plot for WCE image A-D
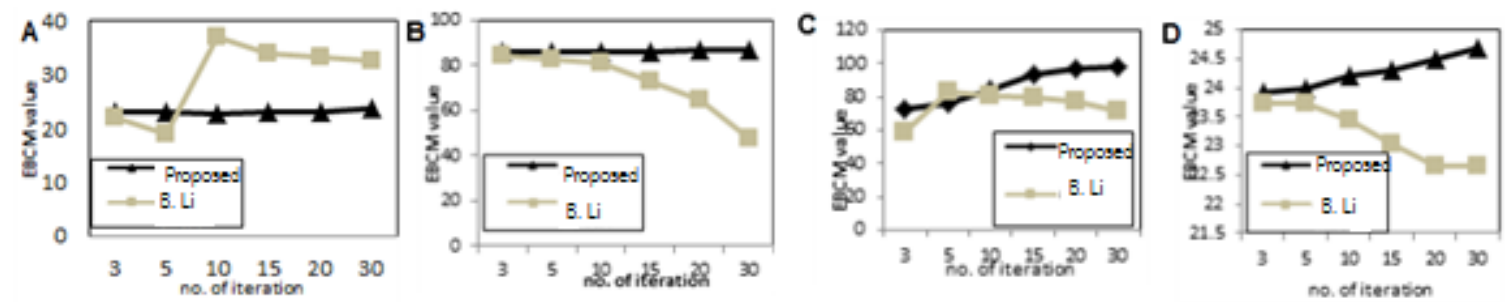

Figure 4. EBCM measure plot for WCE image A-D

Histogram is used to see the distribution of color in an image in red, green and blue channel. Two criteria can be identified in histogram either the image is underexposure or lack of contrast. By using histogram, the characteristics of an image can be detected such as either an image is saturation, spikes and gaps and also provide the information about contrast, sharp and others to interpret the visual of image. Figure 5 shows the histogram for image A-D in red, green and blue channel. Distribution number of pixel can be seen from these histograms at each channel level. 


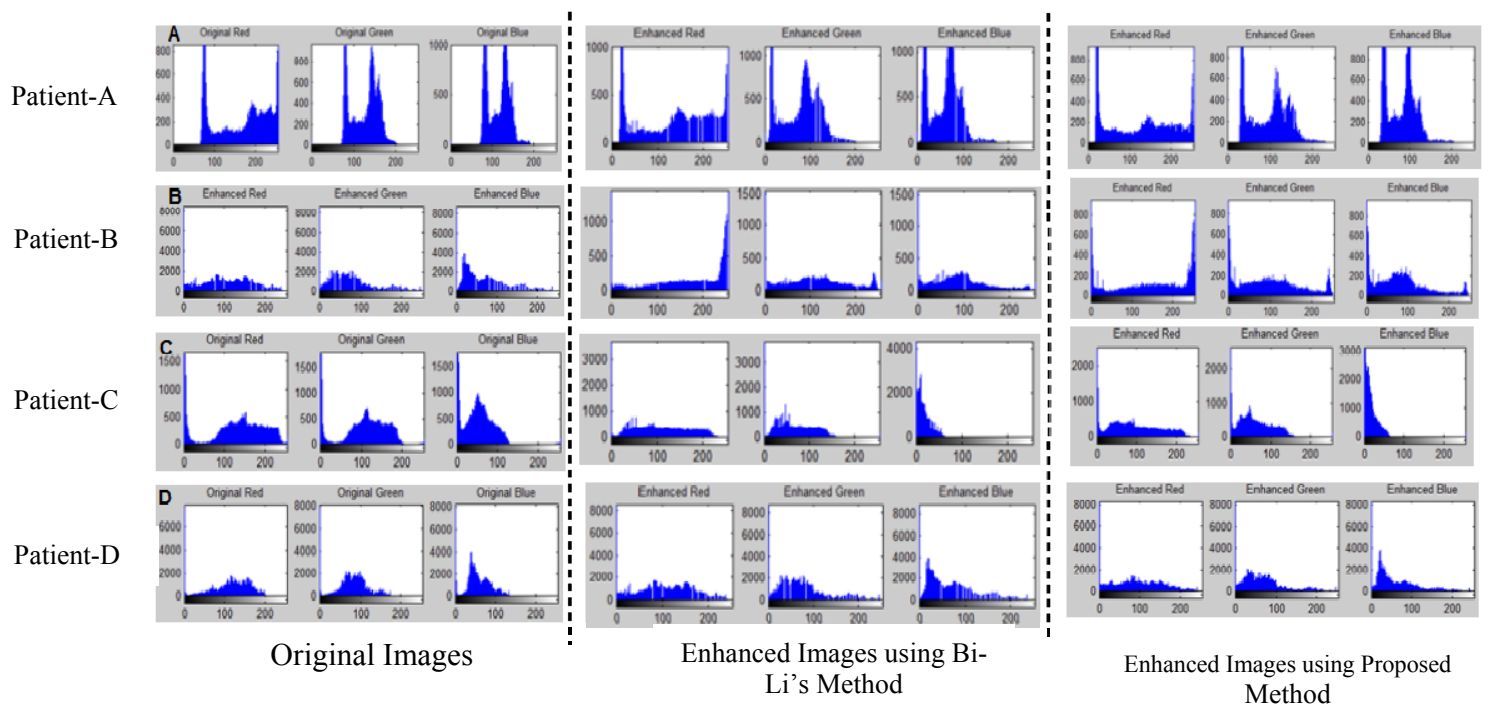

Figure 5. The graph demonstrates the histogram color distribution in red, green and blue channel in WCE images A-D

As can see from Figure 5, the histograms for original image are not distributed over the entire intensity range. Thus, original image has low contrast and vague. Compare to histograms for enhanced images using B. Li's method are wider intensity range and the contrast in the image is increased. The good quality of the image should has wider intensity range which is the pixels should be distributed evenly over the whole intensity rrange and peak of intensity range is higher that means the image is more sharp. Thus, this characteritics is showed in histogram for enhanced image using our proposed method which is better than histogram for enhanced images using B. Li's method.

PSNR of original and enhanced image obtained by B. Li's method and proposed method is presented in Table 1. As we can see from this table, PSNR value of our proposed method is better than B. Li's method. Table 2 shows the measurement of sharpness in WCE image using gradient which is measuring edge information. Let say $\mathrm{I}(\mathrm{x}, \mathrm{y})$ is an image, then the gradient vector is defined as

$$
\nabla I=\left(\frac{d x}{d x} \frac{g r}{d y}\right)
$$

where the partial derivative with respect to $x$ and $y$ is defined as

$$
\begin{aligned}
& \frac{\partial I(x, y)}{\partial x} \approx \frac{I(x+1, y)-I(x-1, y)}{2} \\
& \frac{\partial I(x, y)}{\partial y} \approx \frac{I(x, y+1)-I(x, y-1)}{2}
\end{aligned}
$$

The result proved that both proposed contrast enhancement algorithm and sharpening WCE image algorithm provide better performance compared with B. Li's algorithm since SDME and EBCM value is stable whenever number of iterations increases, and sharpness measurement using gradient and PSNR are both improved by $31.5 \%$ and $20.3 \%$, respectively.

Table 1. PSNR value for enhanced image using B. Li's method and proposed method (in decibel)

\begin{tabular}{ccc}
\hline Image & B.Li's & Proposed \\
\hline Patient A & 15.59504 & 16.18249 \\
Patient B & 16.5804 & 17.45708 \\
Patient C & 14.95192 & 17.98542 \\
Patient D & 22.47994 & 22.57574 \\
\hline
\end{tabular}


Table 2. Sharpness measurements for enhanced image using B. Li's method, and proposed method when number of iteration is 3

\begin{tabular}{ccc}
\hline Image & B.Li's & Proposed \\
\hline Patient A & 5.17439 & 6.14052 \\
Patient B & 5.89158 & 7.70441 \\
Patient C & 3.57323 & 3.70399 \\
Patient D & 2.38227 & 2.42673 \\
\hline
\end{tabular}

\section{CONCLUSION}

Pre-processing technique is a very important process in CAD system before diagnosing a WCE images in order to enhance low quality WCE image, which is noisy, dark and vague or uncertain texture in an image. The aim of pre-processing technique is to simplify a classification task in CAD system. We introduced variance value in anisotropic contrast diffusion to overcome the drawback of original algorithm. Then, in order to get more sharpness in the images, DCT is used which is shown to be effective in enhancing the WCE images. The results proved that the proposed method is better than B. Li's method. Future research work should focus on how to normalize the color WCE image, since abnormalities such as bleeding color has various red colors in order to ease classification task in CAD system.

\section{ACKNOWLEDGEMENTS}

This research is partially supported by University Research Research Grant of Universiti Teknologi Malaysia GUP Tier 1 with Vote No. 05H61 of Ministry of Higher Education (MOHE) year 2014 to 2016.

\section{REFERENCES}

[1] B. Li and M. Q. H. Meng, "Wireless Capsule Endoscopy Images Enhancement Via Adaptive Contrast Diffusion," Journal of Visual Communication and. Image Representation, vol/issue: 23(1), pp. 222-228, 2012.

[2] R. Shahril, et al., "Anisotropic Contrast Diffusion Enhancement Using Variance For Wireless Capsule Endoscopy Images," International Conference on in Informatics, Electronics \& Vision (ICIEV), pp. 1-6, 2014.

[3] Y. G. Lee and G. Yoon, "Bleeding Detection Algorithm For Capsule Endoscopy," World Academy of Science Engineering and Technology, vol/issue: 5(9), pp. 544-549, 2011.

[4] L. Cui, et al., "Bleeding Detection In Wireless Capsule Endoscopy Images By Support Vector Classifier," IEEE International Conference on Information and Automation, pp. 1746-1751, 2010.

[5] P. Szczypiński, et al., "Texture And Color Based Image Segmentation And Pathology Detection In Capsule Endoscopy Videos," Computer Methods and Programs in Biomedicine, vol/issue: 113(1), pp. 396-411, 2014.

[6] T. Ghosh, et al., "An Automatic Bleeding Detection Scheme In Wireless Capsule Endoscopy Based On Histogram Of An RGB-Indexed Image," 36th Annual International Conferene of the IEEE Engineering in Medicine and Biology Society (EMBC), pp. 4683-4686, 2014.

[7] T. Ghosh, et al., "Statistical Feature Based Novel Method To Detect Bleeding In Wireless Capsule Endoscopy Images," International Conference on Informatics, Electronics \& Vision, pp. 1-4, 2014.

[8] G. Pan, et al., "Bleeding Detection In Wireless Capsule Endoscopy Based On Probabilistic Neural Network. Journal of Medical Systems, vol/issue: 35(6), pp. 1477-1484, 2011.

[9] I. N. Figueiredo, et al., "Computer-Assisted Bleeding Detection In Wireless Capsule Endoscopy Images," Journal Computer Methods in Biomechanics and Biomedical Engineering: Imaging \& Visualization, vol/issue: 1(4), pp. 198-210, 2013.

[10] S. Sainju, et al., "Bleeding Detection In Wireless Capsule Endoscopy Based On Color Features From Histogram Probability," 26th IEEE Canadian Conference of Electrical and Computer Engineering, pp. 1-4, 2013.

[11] B. Juntao, "An Improved Medical DR Image Enhancement Method," TELKOMNIKA Indonesian Journal of Electrical Engineering, vol/issue: 12(4), pp. 2718-2723, 2014.

[12] M. Sheng, et al., "Underwater Images Enhancement using Multi-Wavelet Transform and Median Filter," TELKOMNIKA Indonesian Journal of Electrical Engineering, vol/issue: 12(3), pp. 2306-2313, 2014.

[13] P. Perona, and J. Malik, "Scale-Space And Edge Detection Using Anisotropic Diffusion," IEEE Transactions on PAMI 12, pp. 629-639, 1990.

[14] G. Gerig, et al., "Nonlinear Anisotropic Filtering of MRI Data," IEEE Transactions on Medical Imaging, vol/issue: $1(2), 1992$.

[15] R. A. Carmona and S. F. Zhong, "Adaptive smoothing respecting feature directions," IEEE Transactions on Image Processing, vol/issue: 7(3), pp. 353-358, 1998.

[16] R. Shahril, et al., "Pre-Processing Technique Based On Discrete Cosine Transform (DCT) and Anisotropic Contrast Diffusion for Wireless Capsule Endoscopy Images", IEEE Conference on in Biomedical Engineering and Sciences (IECBES, pp. 922-927, 2014

[17] N. Ahmed, et al., "Discrete Cosine Transform," IEEE Transactions on Computers, pp. 90-93, 1973.

[18] A. K. Jain, "Fundamentals of digital image processing," Prentice Hall, 1989. 
[19] A. Beghdadi and L. Negrate, "Contrast enhancement technique based on local detection of edges," Comput. Vis. Graphics Image Process, vol. 46, pp. 162-174, 1989.

[20] T. Celik and T. Tjahjadi "Automatic Image Equalization and Contrast Enhancement Using Gaussian Mixture Modeling," IEEE Transactions on Image Processing, vol/issue: 21(1), 2012.

[21] K. Panetta, et al., "Nonlinear Unsharp Masking for Mammogram Enhancement," IEEE Transactions on Information Technology on Biomedicine, vol/issue: 15(6), 2011.

\section{BIOGRAPHIES OF AUTHORS}

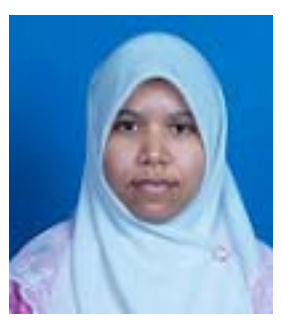

Rosdiana Shahril has received her Bachelor in Computer Engineering from Faculty of Electrical Engineering, Universiti Teknologi Malaysia in 2008. She has completed her Masters in Mathematics (Numerical methods and multigrid) from Faculty of Science, Universiti Teknologi Malaysia in 2012. She worked as a Research Assistant and Analyst Developer for four years. Her current research intererest include mathematical methods, calculus, medical image processing and neural network.

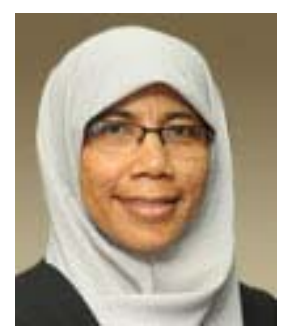

Sabariah Baharun received her Bachelor in Mathematics in August 1982 from Indiana State University, Indiana, USA. She has completed her Masters in Mathematics in 1983 from Ohio University, Ohio, USA. She has obtained her PhD in Applied Mathematics from Universiti Teknologi Malaysia, 2005. Earlier, Dr. Sabariah worked as a Senior Lecturer in the Department of Mathematics under Faculty of Science at Universiti Teknologi Malaysia. She has also served as the Deputy Dean (Academic) in Malaysia-Japan International Institute of Technology, Universiti Teknologi Malaysia for over two years. Currently, Dr. Sabariah is the Associate Proffesor at Malaysia-Japan International Institute of Technology, Universiti Teknologi Malaysia. Her research area in Graph and Fuzzy Graph Modeling, Mathematical Thinking, and wireless communication. She has over 50 Int'l Publication.

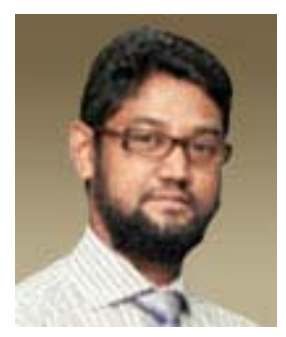

A.K.M. Muzahidul Islam has received M.Sc. in Computer Science and Engineering from Kharkiv National University of Radio Electronics, Ukraine, in 1999 and D.Eng. in Computer Science and Engineering from Nagoya Institute of Technology, Japan in 2007. Dr. Muzahid has recieved Japanese Government Monbusho Scholarship (October 2002 - March 2006). He has worked in various industries in Bangladesh and currently serving as a Senior Lecturer at Malaysia-Japan International Institute of Technology (MJIT) of Universiti Teknologi Malaysia (UTM), Kuala Lumpur. His research interests include Network Achitecture, Communication Protocol, Cognitive Radio Network, Wireless Sensor Network, and Network Security. Dr. Muzahid has published over 60 international research publications. He has served in the 7TH AUN/SEED-Net 2014 Int'l Conference on EEE, ICBAPS2015, ICIEV15 and ICAEE 2015 Int'1 Conferences. Currently is serving as the Program Chair of ICAICT 2016 and Secretariat for the ICaTAS 2016. Dr. Muzahid is a Senior IEEE Member (SMIEEE) and is an IET Member with Designatory Letters (MIET). 